\title{
AVALIAÇÃO DE COR DE DIFERENTES MARCAS E SISTEMAS DE RESINA COMPOSTA POR ESPECTROFOTOMETRIA
}

\author{
COLOR EVALUATION OF DIFFERENT BRANDS AND \\ COMPOSITE RESIN SYSTEMS BY SPECTROPHOTOMETER
}

\author{
Indiara Migot', Ana Bárbara Marques ${ }^{2}$, Isadora Rocha ${ }^{3}$, Andrea Cavalcanti ${ }^{4}$, Saryta Argolo ${ }^{5}$ \\ 'Cirurgiã-Dentista, Graduada pela Faculdade Independente do Nordeste. Vitória da Conquista, Bahia, Brasil. indiara70@hotmail.com \\ ${ }^{2}$ Cirurgiã-Dentista, Graduada pela Faculdade Independente do Nordeste. Vitória da Conquista, Bahia, Brasil. anabarbara.mv@hotmail.com \\ ${ }^{3}$ Graduação em andamento na Faculdade Independente do Nordeste. Vitória da Conquista, Bahia, Brasil. isadorarochagbi@hotmail.com \\ ${ }^{4}$ Doutora em Clínica Odontológica. Professora na Universidade Federal da Bahia. Salvador, Bahia, Brasil. dea.cavalcanti@uol.com.br \\ ${ }^{5}$ Mestre em Odontologia. Professora na Faculdade Independente do Nordeste. Vitória da Conquista, Bahia, Brasil. saryta_argolo@hotmail.com
}

\begin{abstract}
RESUMO | Objetivo: avaliar a cor de três resinas compostas de cor A2 e A3 de diferentes marcas comerciais, através da análise por espectrofotômetro VITA Easyshade ${ }^{\circledR}$ Compact. Método: Para a análise de cor, foram confeccionados 36 corpos de prova $(n=6)$, em formato de disco, a partir de uma matriz de aço. Para cada espécime, foi realizado 5 aferições e os valores de $L^{*}, a^{*}, b^{*}$ e L*, $C^{*}, h^{*}$ anotados. Resultados: Verificou-se que a cor $\mathrm{A} 2$ diferiu estatisticamente da fornecida pela escala vita $(p<0,0001)$, e diferenças estatísticas entre as resinas compostas não foram observadas $(p=0,56)$. As resinas compostas Opallis e Fill Magic de cor A3 diferiram estatisticamente da fornecida pela escala vita $(p<0,0001)$, porém $\circ$ mesmo não foi observado com a resina composta Filtek Z250 e diferenças entre as resinas compostas foram observadas $(p=0,56)$, sendo a resina composta Opallis e Fill Magic semelhantes entre si e estatisticamente diferentes da Filtek Z250. Conclusão: Conclui-se que a maioria das resinas compostas estudadas não apresentou correspondência de cor positiva com a escala de referência VITA Easyshade ${ }^{\circledR}$ Compact, o que dificulta a execução do profissional durante procedimentos restauradores estéticos.
\end{abstract}

Palavras-chave: estética dentária, cor, resinas compostas

\begin{abstract}
Purpose: to evaluate the three color composites of $A 2$ and $A 3$ color of different trademarks, through the review by VITA Easyshade ${ }^{\circledR}$ Compact spectrophotometer. Method: for the analysis of color, 36 specimens were made $(n=6)$, disk-shaped, from an array of steel. For each specimen, was held 5 measurements and the values of $L^{*}, a^{*}, b^{*}$ e $L^{*}, C^{*}, h^{*}$. Results: It was found that the color A2 differed statistically from the provided by vita $(p<0.0001)$, and statistical differences between the composite resins were not observed $(p=0.56)$. The composite resins Opallis and Fill Magic of color A3 differed statistically from the provided by vita $(p<0.0001)$, but the same was not observed with the composite resin Filtek Z250 and differences between the composite resins were observed $(p=0.56)$, being the composite resin Opallis and Fill Magic similar to each other and statistically different from the Filtek Z250. Conclusion: it is concluded that the majority of composite resins studied showed no color matching well with the scale of referencia VITA Easyshade ${ }^{\circledR}$ Compact, which makes the implementation of professional during procedures aesthetic restorative.
\end{abstract}

Keywords: dental esthetics, color, Composite Resins 


\section{INTRODUÇÃO}

A busca constante por materiais restauradores com a finalidade de reproduzir a estrutura dental de forma biomimética, deve-se à grande exigência e expectativa dos pacientes que estão cada vez mais procurando por tratamentos estéticos'. A Odontologia vem ao longo dos tempos travando uma luta incansável pela descoberta de um material restaurador que consiga além de restabelecer a função do elemento dental, reproduzir de uma forma natural, a cor da estrutura dentária ${ }^{2}$.

Devido os conhecimentos atuais sobre adesão, a filosofia restauradora se modificou. A resina composta de inserção direta é considerada o material restaurador de primeira escolha, devido à sua característica de reforço do remanescente dental, possibilidade de confecção de preparos conservadores, ampla disponibilidade de cores, e a sua alta capacidade de simulação das características ópticas do dente natural ${ }^{3}$. Diante disso, a seleção da cor e do material restaurador utilizado é um passo clínico importante e deve ser realizada de maneira criteriosa.

Tradicionalmente, a seleção da cor das resinas compostas é realizada por métodos visuais através de comparações com escalas de cor de referência ${ }^{4}$. Porém, este método apresenta limitações como a incapacidade de reproduzir fielmente as cores dos dentes naturais, uma vez que os dentes da escala são confeccionados com materiais diferentes dos produtos restauradores e a falta de padronização das marcas tanto para os fabricantes dos materiais restauradores quanto para os das escalas 5 .

Com o avanço da tecnologia, foi desenvolvido a técnica de seleção de cor que faz uso de instrumentos eletrônicos, obtendo assim, medidas mais objetivas e sem influência do operador, do ambiente e da iluminação $0^{6,7}$. $O$ espectrofotômetro é um aparelho que foi criado para uso odontológico como objetivo de minimizar a subjetividade da estimativa visual da cor, sendo capaz de detectar pequenas diferenças na cor, que a olho humano não são captadas ${ }^{8}$. Tem como função calcular a cor a partir da mensuração da quantidade, da intensidade e da composição espectral da luz absorvida, transmitida ou refletida em todos os comprimentos de onda visíveis após a interação com a superfície do objeto, podendo este ser dentes vitais ou extraídos e materiais restauradores ${ }^{5,9}$.

A seleção de cores desses equipamentos é baseada na extrapolação dos valores de mensuração dentre os valores mais significativos para o clínico, tais como as referências de cores dos sistemas de escalas?. A precisão na seleção da cor pode ser afetada caso seja feito por equipamentos diferentes, pois a concordância entre eles é relativamente fraca em comparação com a confiabilidade de mensuração realizada por um mesmo instrumento, - que pode dificultar a chegada de uma cor final ou a comunicação entre o cirurgião e o técnico laboratorial $^{10}$.

O objetivo do presente estudo é avaliar a cor de três resinas compostas de cor A2 e A3 de diferentes marcas comerciais, através da análise no espectrofotômetro VITA Easyshade ${ }^{\circledR}$ Compact.

\section{MATERIAIS E MÉTODOS}

Para a confecção dos corpos de prova $(n=36)$ foi utilizado resinas compostas de cor A2 e A3 de diferentes marcas comerciais (Quadrol): Opallis@(FGM, Joinville, SC, Brasil), Filtek Z250 XT (3MEspe, St. Paul, EUA) e Fill Magic@(Vigodent, Rio de Janeiro, RJ, Brasil).

As amostras foram confeccionadas com auxílio de uma matriz de aço retangular contendo 01 orifício de $8 \mathrm{~mm}$ de diâmetro por $2 \mathrm{~mm}$ de altura. A matriz de aço foi apoiada sob uma placa de vidro e, a seguir, dois incrementos de resina composta foram inseridos utilizando a técnica incremental. O compósito foi pressionado com auxílio de uma tira de poliéster (Maquira@, Maringá, PR, Brasil) e uma lamínula de vidro, para permitir o escoamento do excesso de material. A resina composta foi fotoativada sob a matriz de poliéster e lamínula de vidro durante 30 segundos, utilizando um fotopolimerizador de LED 


\begin{tabular}{|c|c|c|c|c|}
\hline $\begin{array}{l}\text { MARCA } \\
\text { COMERCIAL } \\
\text { (Fabricante) }\end{array}$ & COR & COMPOSIÇÃO & TIPO & LOTE \\
\hline Opallis ${ }^{\ominus}$ (FGM) & A2 & $\begin{array}{l}\text { Nano-híbrida, Bis-GMA, } \\
\text { Bis-EMA, UDMA, } \\
\text { TEGDMA, cargas de } \\
\text { vidro de Bário-Aluminio, } \\
\text { silicato silanizados com } \\
\text { nanopartículas de dióxido } \\
\text { de silíio e } \\
\text { canforoquinona como } \\
\text { fotoiniciador. }\end{array}$ & Esmalte & 121214 \\
\hline $\begin{array}{l}\text { FiltekTM }{ }^{M} 250 \\
X T \text { (3M ESPE) }\end{array}$ & A2 & $\begin{array}{c}\text { Micro-híbrida, } \\
\text { zircônia/sílica } \\
\text { (cerâmica/silano), Bìs- } \\
\text { GMA, Bis-EMA, UDMA, } \\
\text { PEGDMA e TEGDMA. }\end{array}$ & Universal & 123907 \\
\hline $\begin{array}{l}\text { Fill Magic }^{\odot} \\
\text { (Vigodent) }\end{array}$ & A2 & $\begin{array}{c}\text { Híbrido de partículas } \\
\text { finas, Bis-GMA, Bis-EMA, } \\
\text { UDMA e TEGMA. }\end{array}$ & Esmalte & 1403184 \\
\hline Opallis ${ }^{\odot}(F G M)$ & A3 & $\begin{array}{l}\text { Nano-híbrida, Bis-GMA, } \\
\text { Bis-EMA, UDMA, } \\
\text { TEGDMA, cargas de } \\
\text { vidro de Bário-Aluminio, } \\
\text { silicato silanizados com } \\
\text { nanopartículas de dióxido } \\
\text { de silíicio e } \\
\text { canforoquinona como } \\
\text { fotoiniciador. }\end{array}$ & Esmalte & 291014 \\
\hline $\begin{array}{l}\text { FiltekTM Z250 } \\
\text { XT (3M ESPE) }\end{array}$ & A3 & $\begin{array}{c}\text { Micro-híbrida, } \\
\text { zircônia/sílica } \\
\text { (cerâmica/silano), Bis- } \\
\text { GMA, Bis-EMA, UDMA, } \\
\text { PEGDMA e TEGDMA }\end{array}$ & Universal & 163574 \\
\hline $\begin{array}{l}\text { Fill Magic }{ }^{\odot} \\
\text { (Vigodent) }\end{array}$ & A3 & $\begin{array}{c}\text { Híbrido de partículas } \\
\text { finas, Bis-GMA, Bis-EMA, } \\
\text { UDMA e TEGMA. }\end{array}$ & Esmalte & 1403494 \\
\hline
\end{tabular}

Quadro I. Composição básica e especificações técnicas das resinas compostas utilizadas no estudo.

(Radii Cal@ SDI, Victoria, Austrália), com densidade de potência de aproximadamente $1100 \mathrm{~mW} / \mathrm{cm} 2$.

Os corpos de prova foram removidos do molde e o outro lado do disco foi polimerizado por mais 30 segundos.

Após a fotoativação, os discos de resina foram imersos em água destilada, visando à remoção da camada superficial de resina composta não polimerizada pelo contato com o oxigênio. Em seguida, foram armazenados individualmente em recipientes devidamente vedados para proteção de exposição luminosa durante 48 horas, antes de receberem o acabamento e polimento superficial.

Após 48 horas de armazenamento em água, os corpos de prova foram removidos individualmente com o auxílio de uma pinça clínica e secos em papel absorvente e a remoção dos excessos da resina composta com a lixa de óxido de alumínio de numeração \#180 (Carborundum $@$, São Paulo, SP, 
Brasil) foi realizada. Para a etapa de acabamento, foi utilizado o disco de lixa (TDV@, Pomerode, SC, Brasil) acoplado em mandril em baixa rotação, em sequência decrescente de abrasividade (granulosidade média, fina e extrafina), durante 10 segundos para cada lado do espécime. $O$ polimento foi realizado com disco de feltro (TDV@, Pomerode, SC, Brasil) e pasta de polimento Diamond R (FGMC, Joinville, SC, Brasil).

Depois de polidos, os espécimes foram lavados e secos cuidadosamente com papel absorvente para então serem submetidos à análise de cor inicial. A análise dos corpos de prova e da palheta da escala Vita Classical@ (Vita, Zahnfabrik, Sackingen, Alemanha) foi realizada com o espectrofotômetro (Vita EasyShade ${ }^{\circledR}$, Vident, Brea, CA, EUA) com padrão de observação para o sistema CIELAB.

O aparelho foi constantemente calibrado durante as mensurações de acordo com as recomendações do fabricante. As mensurações foram realizadas com a ponta do instrumento posicionada no centro do corpo de prova, o qual estava posicionado no dispositivo de teflon branco opaco com um orifício central de $8 \mathrm{~mm} \times 2 \mathrm{~mm}$, confeccionado para uniformizar as condições de análise. Para cada espécime, a medição foi repetida cinco vezes e os valores de $L^{*}$ indica luminosidade, $a^{*}$ representa a matriz no eixo vermelho-verde, $b^{*}$ significa $a$ matriz no eixo amarelo-azul e $L^{*}, C^{*}, h^{*}$ obtidos e anotados.
Para a determinação e avaliação quantitativa da correspondência de cor, foi utilizado o critério recomendado pela Comissão Internacional em Iluminação (Commission Internationale de l'Eclairage - $\mathrm{CIE})^{11}$, segundo a qual correspondência/diferença de cores pode ser expressa matematicamente pelo valor de $\Delta \mathrm{E}$, que define a diferença total da cor da amostra em relação ao padrão e, é calculado através da seguinte fórmula: $\Delta \mathrm{E} 2=[(\Delta \mathrm{L}) 2+(\Delta \mathrm{c}) 2+$ $(\Delta h) 2]$.

\section{ANÁLISE ESTATÍSTICA}

Inicialmente foi realizada a análise exploratória dos dados para verificar a homogeneidade das variâncias e para determinar se os erros experimentais apresentavam distribuição normal. A análise estatística inferencial foi feita no programa estatístico Bioestat, versão 5.3, com nível de significância de $5 \%$.

Para comparação entre a cor da resina composta de diferentes fabricantes e a fornecida pela escala vita, foi utilizado o teste $t$ de Student para 1 -amostra.

Para comparação entre as cores fornecidas por resinas compostas de três diferentes fabricantes em dois níveis (A2 e A3), foi aplicada a Análise de Variância (ANOVA) a 1 critério.

\section{RESULTADOS}

As tabelas 1 e 2 apresentam à média e desvio-padrão das cores fornecidas pelos diferentes fabricantes (A2 e A3).

De acordo com a análise destes dados, verifica-se que a cor A2 de todas as resinas compostas testadas diferiram estatisticamente da fornecida pela escala vita (teste $t$ de Student para 1 amostra; $p<0,0001$ ). Entretanto, diferenças estatísticas entre as resinas compostas não foram observadas (ANOVA 1 critério; $p=0,56)$.

De acordo com a análise destes dados, verifica-se que a cor A3 das resinas compostas Opallis e Fill Magic diferiu estatisticamente da fornecida pela escala vita (teste $t$ de Student para 1 amostra; $p<0,0001$ ), porém - mesmo não foi observado na Z250. Diferenças entre as resinas compostas foram observadas (ANOVA 1 critério; $p=0,01$ ), sendo Opallis e Fill Magic semelhantes entre si e estatisticamente diferentes da Z250. 
Tabela 1. Média (desvio-padrão) dos valores de $\Delta \mathrm{E}$ da cor $\mathrm{A} 2$ das resinas compostas estudadas e valor de $\Delta \mathrm{E}$ da Escala Vita Classical.

\begin{tabular}{ccc}
\hline & A2 & Escala Vita Classical \\
\hline Opallis & $64,68(12,60) \mathrm{A}^{*}$ & 19,89 \\
Fill Magic & $73,06(13,15) \mathrm{A}^{*}$ & \\
Z250 & $73,34(16,43) \mathrm{A}^{*}$ & \\
\hline
\end{tabular}

*Médias seguidas por letras distintas representam significância estatística $(p<0,05)$.

* Maiúsculas comparam as resinas compostas e asteriscos representam valores estatisticamente diferentes da escala vita.

Tabela 2. Média (desvio-padrão) dos valores de $\Delta \mathrm{E}$ da cor $\mathrm{A} 3$ das resinas compostas estudadas e valor de $\Delta E$ da Escala Vita Classical.

\begin{tabular}{ccc}
\hline & A3 & Escala Vita Classical \\
\hline Opallis & $122,67(44,10) \mathrm{A}^{*}$ & 39,89 \\
Fill Magic & $91,15(18,68) \mathrm{A}^{*}$ & \\
Z250 & $39,25(5,12) \mathrm{B}$ \\
*Médias seguidas por letras distintas representam significância estatística $(\mathrm{p}<0,05)$.
\end{tabular}

* Maiúsculas comparam as resinas compostas e asteriscos representam valores estatisticamente diferentes da escala vita.

\section{DISCUSSÃO}

Não há dúvidas de que a cor do material é altamente importante para $\circ$ sucesso de uma restauração com resina composta, sendo, portanto, amplamente estudada por diversos autores ${ }^{12}$. A seleção de cor muitas vezes é negligenciada por erro ou imperícia do cirurgião-dentista, o que resulta no comprometimento negativo deste passo ${ }^{12}$ importante para o sucesso clínico da restauração. Para garantir êxito, o momento da escolha do material é fundamental, assim como a estabilidade de cor e as demais propriedades físicas e químicas do material resinoso 6 .

No presente estudo, foi utilizado o espectrofotômetro para avaliação de cor das resinas compostas avaliadas. Segundo Alves ${ }^{6}$ em 2014, a técnica de seleção de cor através de aparelhos como espectrofotômetros, deve ser a mais recomendada pelos profissionais, por tornar esse processo objetivo, quantificável e rápido, já que cada vez mais, tem-se a necessidade de realizar procedimentos estéticos perfeitos e sem diferença de cor entre o elemento dental e o material.

Para estudos sobre a estabilidade de cor das resinas compostas com o uso destes aparelhos, temse utilizado o sistema CIE L*, a* e b. Este sistema consiste em parâmetros em que $\circ L^{*}$ se refere às coordenadas relacionadas com a cor nas axiais vermelho-verde e amarelo-azul, respectivamente ${ }^{13}$. Desta maneira torna-se possível realizar comparações entre duas mensurações utilizando as leituras de "L*", "a*" e "b*" de cada medida, por meio de fórmulas pré-definidas, tendo em números as diferenças das cores entre as duas mensurações, apresentadas através de $\Delta \mathrm{E}^{9,14}$.

O presente estudo avaliou as hipóteses experimentais de que as resinas estudadas iriam se assemelhar à escala de referência em relação à cor. De acordo com os resultados obtidos, observa-se que para os grupos de cor A2, todas as resinas compostas diferiram estaticamente em relação à cor quando comparadas com escala de referência. Já para os 
grupos de cor A3, apenas a resina composta Filtek Z250 apresentou similaridade de cor com a escala. Esta não compatibilidade de cor entre as resinas compostas avaliadas e a escala Vita Classical $\mathbb{R}$ podem estar relacionadas com alguns fatores.

A avaliação de cor para as resinas compostas utilizadas neste estudo adotou o padrão $A B C D$ da escala de cores Vita Classical ${ }^{\circledR}$ como referência para classificação da tonalidade de seus materiais restauradores ${ }^{7,15}$. Uma observação importante é que a escala utilizada neste estudo, a escala de referência para escolha de cor em restaurações com resina composta, é confeccionada em cerâmica, ou seja, de material distinto e, portanto, apresentam interações diferentes com a luz ${ }^{14,16}$.

De modo semelhante, Alves et al ${ }^{6}$ em 2014 avaliaram se 0 espectrofotômetro VITA Easyshade $\AA$ Compact faz leituras semelhantes de cor da resina composta Filtek ${ }^{\circledR}$ Z350. Os autores verificaram também se a hidratação do compósito influencia nestas leituras e se há diferença de cor entre a resina composta utilizada e a escala Vita Classical ${ }^{16}$. Para o estudo, os autores confeccionaram 10 corpos de prova da resina composta na cor $A 2$. As coordenadas de cor do sistema CIELAB dos corpos de prova e da cor A2 da escala Vita Classical foram aferidas no espectrofotômetro para calcular a diferença de cor $(\Delta \mathrm{E})$ entre o corpo de prova e escala Vita Classical. Os autores observaram diferenças significantes entre os modos de leitura em ambas às condições (inicial e $24 \mathrm{~h}$ ) e entre os períodos de hidratação (inicial e $24 \mathrm{~h}$ ) também, observando-se valores menores de $\Delta \mathrm{E}$ após hidratação. Diante dos resultados, concluíram a cor $A 2$ da resina Filtek ${ }^{\circledR} Z 350$ não correspondeu à cor A2 da escala Vita Classical.

De maneira controversa, Freitas et $\mathrm{al}^{16}$, em 2008 avaliaram a eficiência na escolha de cor entre duas escala de cor, Vitapan Classical Vita e Vita 3D-Master. Foram escolhidos quatro dentes hígidos, ânterosuperiores, de dois voluntários, um incisivo central superior esquerdo e um canino superior esquerdo em cada voluntário, o critério de seleção dos dentes foi subjetivo e fundamentou-se nos que possuíam cores com alguma similaridade com as escalas Vitapan Classical e Vitapan 3D-Master. A seleção foi pelo método visual, pois se trata do utilizado na rotina clínica. A cor dos elementos dentários foi escolhida por 3 profissionais com experiência em prótese dentária. Foram entrevistados 30 profissionais e 15 estudantes de Odontologia, foi aplicado um questionário com perguntas sobre seleção de cor, os dados obtidos foram submetidos ao teste não paramétrico de Man-Whitney (5\%), os resultados mostraram que não existe diferença estatística entre as escalas quando a seleção de cor é feita de maneira adequada, sendo então, necessário - aprimoramento técnico da seleção de cor pelos cirurgiões dentistas e acadêmicos.

Idealmente, uma resina composta classificada por meio de uma escala de cor deve coincidir com resinas compostas da mesma e de outras marcas comerciais ${ }^{17}$. Quando os compósitos foram analisados entre si, diferenças entre as resinas compostas foram observadas, sendo Opallis e Fill Magic semelhantes entre si e estatisticamente diferentes da Filtek Z250. Uma hipótese para esta diferença deve-se a composição e características dos materiais restauradores ${ }^{18,19}$.

As resinas compostas testadas na presente investigação diferem quanto ao tipo do tamanho das partículas de carga da matriz inorgânica ${ }^{20}$.

Segundo Buchalla et al, $2002^{19}$ quanto menor o tamanho das partículas, menor será a estabilidade de cor da resina composta. As resinas compostas Opallis@ são, entre as estudadas, as que apresentam - menor tamanho de carga $^{20}$ das partículas nanohíbrida, quando comparadas as demais do estudo.

A quantidade e composição do agente fotoiniciador de polimerização é outro fator que pode alterar a cor de um material resinoso ${ }^{20}$. Atualmente, o agente fotoiniciador mais utilizado é a canforoquinona. Apesar de ser utilizada em baixas concentrações na grande maioria das resinas compostas, este agente influência de forma significativa a cor do material $^{14,20}$. Este é um composto químico de cor amarela, que durante a fotoativação muda para uma cor transparente. Logo, se esta fotopolimerização não for suficiente, certa quantidade da cor amarela ainda permanecerá no material. Portanto, os materiais resinosos que possuírem como agente fotoativador a canforoquinona devem ser feita a polimerização corretamente, respeitando o tempo 
de fotoativação recomendado pelo fabricante e quantidade de emissão de luz para adequada conversão monomérica em polímeros, assim, o material tem menos probabilidade de reter a cor amarelada $^{20}$.

Segundo, Baratieri ${ }^{4}$ (2008) a presença de água em qualquer objeto modifica sua interação com a luz, já que o índice de refração da luz é 30\% maior na água do que no ar, portanto, a maioria das resinas compostas pode mudar de coloração quando hidratada. A teoria da hidratação explica que a absorção de água altera as leituras de cor da resina composta. Os compósitos de características micro e nanoparticuladas apresentam uma maior taxa de sorção de água, fazendo com que estes materiais apresentem menor estabilidade de cor ao longo do tempo ${ }^{20}$.

\section{CONCLUSÃO}

Sem dúvida, a seleção de cor é uma etapa extremamente complexa no processo de tratamento estético restaurador. Tendo em vista os aspectos observados, pode-se concluir que a maioria das resinas compostas estudadas não apresentou correspondência de cor entre si e com a escala de referência Vita Classical, portanto, não se deve considerar viável a utilização ou substituição por resinas de marcas comerciais distintas, assim como, não se pode usar a escala como ferramenta única no processo de seleção da cor de resinas compostas.

\section{CONTRIBUIÇÕES DOS AUTORES}

Migot I, Marques AB participaram da confecção dos corpos e prova e testes de análise de cor e redação do artigo centífico. Rocha I participou da concepção do delineamento da pesquisa, interpretação dos resultados, redação e o encaminhamento do artigo centífico. Cavalcanti A participou da concepção, delineamento, busca e análise estatística dos dados da pesquisa. Argolo $S$ participou da coleta de dados da pesquisa, interpretação dos dados, interpretação dos resultados, redação e encaminhamento do artigo científico.

\section{CONFLITOS DE INTERESSES}

Nenhum conflito financeiro, legal ou político envolvendo terceiros (governo, empresas e fundações privadas, etc.) foi declarado para nenhum aspecto do trabalho submetido (incluindo mas não limitandose a subvenções e financiamentos, conselho consultivo, desenho de estudo, preparação de manuscrito, análise estatística, etc).

\section{REFERÊNCIAS}

1. Dias HB, Carrera ET, de Souza Rastelli AN. The influence of $\mathrm{pH}$ and chemical composition of beverages on color stability of a nanofilled composite resin. Gen Dent. 2016;64(6):21-27.

2. Pereira ASR, Pires MM, Figueiredo JAP, Mota EG, Pires LAG. Análise dos níveis de cinza de 4 resinas compostas microhíbridas utilizando um sistema de radiografia digital direto. Revista Odo Cien. 2005;20(47):3-10.

3. Randolph LD, Palin WM, Leloup G, Leprince JG. Filler characteristics of modern dental resin composites and their influence on physico-mechanical properties. Dent Mater. 2016;32(1 2):1586-1599. doi: 10.1016/i.dental.2016.09.034

4. Baratieri LN. Soluções Clínicas - Fundamentos e Técnicas. 1 ed. Florianópolis: Editora Ponto; 2008.

5. Santos GB, Alto VD, Filho HR, da Silva EM, Fellows CE. Light transmission on dental resin composites. Dent Mater. 2008;24(5):571-576. doi: 10.1016/i.dental.2007.06.015

6. Alves JKG, Aued N, Soares FZM, Jacques LB, Kaizer $M R$, Mallmann A. Avaliação da cor de um compósito com espectrofotômetro em diferentes modos de leitura e condições de armazenagem. Rev RFO. 2014;19(1):101-106.

7. Dantas AAR, Florez FLE, Campos EA, Andrade MF, Saad JRC, Oliveira Júnior $O B$. Correspondência de cor de diferentes marcas e sistemas de resina composta em relação à escala vita classical. Rev Pós Grad. 2011 ; 18(1):45-51.

8. Vichi A, Louca C, Corciolani G, Ferrari M. Color related to ceramic and zirconia restorations: A review. Dent Mater. 2011 ;27(1):97-108. doi: 10.1016/i.dental.2010.10.018

9. Erdemir U, Yildiz E, Eren MM. Effects of sports drinks on color stability of nanofilled and microhybrid composites after longterm immersion. J Dent. 2012;40(2):55-63. doi: 10.1016/i. ident.2012.06.002

10. Dozic' A, Kleverlaan CJ, El-Zohairy A, Feilzer AJ, Khashayar $G$. Performance of Five commercial available tooth colormeasuring devices. J Prosthodont. 2007;16(2):93-100. doi: 10.1111/i.1532-849X.2007.00163.x

11. Commission Internationale de l'Eclairage (CIE). Colorimetry - technical report. 3rd ed. Vienna: Bureau Central de La CIE; 2004. 
12. Russel MD, Gulfraz M, Moss BW. In vivo measurement of colour changes in natural teeth. J Oral Rehabil.

2000;27(9):786-92.

13. Portero PP et al. Análise Instrumental da Correspondência de Cor de Resinas Compostas. Dental science. 2010;3:130-

140.

14. Lee YK, Lim BS, Rhee SH, Yang HC, Lim YK. Changes in scattering and absorption properties of esthetic filling materials after aging. J Biomed Mater Res B Appl Biomater. 2007;80(1):131-9. doi: 10.1002/ibm.b.30577

15. Janda R, Roulet JF, Kaminsky M, Steffin G, Latta M. Color stability of resin matrix restorative materials as a function of the method of light activation. Eur J Oral Sci. 2004;1 12(3):2085. doi: $10.1111 / \mathrm{i} .1600-0722.2004 .00125 . x$

16. Freitas AC, Alves BP, Rodrigues ARM, Junior RRP. Avaliação comparativa entre as escalas de cores Vitapan Classical e 3D-Master. RGO. 2008;56(1):53-57.

17. Kim BJ, Lee YK. Influence of the shade designation on the color difference between the same shade-designated resin composites by the brand. Dent Mater. 2009;25(9):1 1 148-54. doi: 10.1016/i.dental.2009.04.001

18. Douglas RD. Color stability of new-generation indirect resins for prosthodontic application. J Prosthet Dent. 2000;83(2):16670.

19. Buchalla W, Attin T, Hilgers RD, Hellwig E. The effect of water storage and light exposure on the color and translucency of a hybrid and a microfilled composite. J Prostate Dent. 2002;87(3):264-70.

20. Donato LFA, Borges BCD, Vasconcelos AAM, Cruz GFF, Santos AJS, Seabra FRG et al. Avaliação da profundidade de cura de dois compósitos com diferentes tamanhos de partículas. Odontol Clin Cient. 2011 ; 10(3):277-280. 